\title{
Double Contact During Drop Impact on a Solid Under Reduced Air Pressure
}

\author{
Er Qiang Li, ${ }^{1,2}$ Kenneth R. Langley, ${ }^{1}$ Yuan Si Tian, ${ }^{1}$ Peter D. Hicks, ${ }^{3}$ and Sigurdur T. Thoroddsen ${ }^{1, *}$ \\ ${ }^{1}$ Division of Physical Sciences and Engineering, King Abdullah University of Science and Technology (KAUST), \\ Thuwal 23955-6900, Saudi Arabia \\ ${ }^{2}$ Department of Modern Mechanics, University of Science and Technology of China, Hefei 230027, China \\ ${ }^{3}$ School of Engineering, Fraser Noble Building, King's College, University of Aberdeen, Aberdeen AB24 3UE, United Kingdom
}

(Received 25 June 2017; published 20 November 2017)

\begin{abstract}
Drops impacting on solid surfaces entrap small bubbles under their centers, owing to the lubrication pressure which builds up in the thin intervening air layer. We use ultrahigh-speed interference imaging, at 5 Mfps, to investigate how this air layer changes when the ambient air pressure is reduced below atmospheric. Both the radius and the thickness of the air disc become smaller with reduced air pressure. Furthermore, we find the radial extent of the air disc bifurcates, when the compressibility parameter exceeds $\sim 25$. This bifurcation is also imprinted onto some of the impacts, as a double contact. In addition to the central air disc inside the first ring contact, this is immediately followed by a second ring contact, which entraps an outer toroidal strip of air, which contracts into a ring of bubbles. We find this occurs in a regime where Navier slip, due to rarefied gas effects, enhances the rate gas can escape from the path of the droplet.
\end{abstract}

DOI: 10.1103/PhysRevLett.119.214502

Controlled drop impacts on solid surfaces are fundamental to many manufacturing processes, from spray coating to inkjet fabrication of precision electronics and displays $[1,2]$. Entrapment of minuscule air bubbles interferes with the uniformity of coatings and can degrade contact between the liquid and the solid. One possible approach to eliminate such bubbles is to perform the fabrication under vacuum. Herein we show that this is easier said than done. Even though the bubbles become progressively smaller at reduced air pressure, they are never eliminated. Furthermore, at the lowest pressures the number of entrapped bubbles can increase, and in some cases we even observe double circular contacts between the drop and the solid, Fig. 1(a). Such fine interface structures may, for example, interfere with transmission of light for optical components.

The dynamical importance of the surrounding air was demonstrated by $\mathrm{Xu}$ et al. [3], who showed that impact splashing can be suppressed by reducing the air pressure. Mandre et al. [4] proposed to explain this by suggesting the air layer makes the drop skate along the surface without making contact. However, the contraction of the central air disc is driven by capillary-inertial dynamics [5], which assumes contact between the two, long before the emergence of the splashing lamella [6]. Indeed, Driscoll and Nagel [7] verified with interferometry that outside of the central air disc the liquid is in contact with the solid. It is now accepted that the above suppression of splashing is related to the dynamics at the tip of the expanding lamella moving along the substrate, not the central air disc [8,9]. Gliding on the air layer is only observed at very low impact velocities [10,11], or for highly viscous drops, which do not splash [12]. Compliance of the surface has also been found to reduce splashing [13].
Numerous theoretical studies address the formation of a central air disc by balancing lubrication pressure in the air layer and the inviscid inertia within the drop liquid. This is characterized by the Stokes number $\mathrm{St}=\mu_{q} /\left(\rho_{\ell} R_{b} V\right)$,

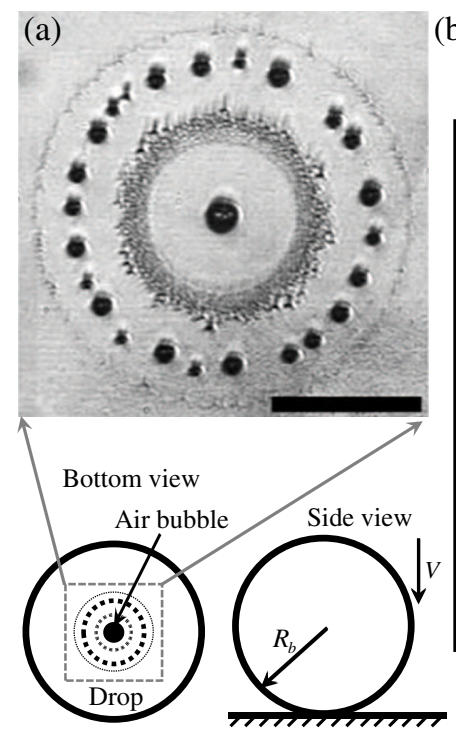

(b)

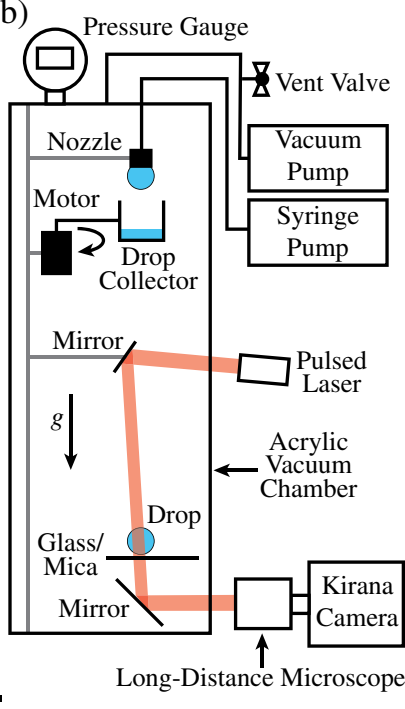

FIG. 1. (a) Microbubble pattern under the center of a drop impacting a freshly cleaved mica surface at reduced air pressure $(P=6.2 \mathrm{kPa}), V=4.94 \mathrm{~m} / \mathrm{s}, R_{b}=3.52 \mathrm{~mm}, \mathrm{St}=1.06 \times 10^{-6}$, and $\epsilon^{-1}=386$. Scale bar is $100 \mu \mathrm{m}$. (b) Sketch of the experimental setup. Drops were formed at an adjustable height nozzle fed by a syringe pump and pinched off by gravity, inside a custommade acrylic vacuum chamber. A drop collector caught drops during depressurization and was rotated out of the drop path to take measurements. The Kirana camera captured interference fringes caused by optical path length differences from light transmission and reflection between the drop interface and glass or mica surface. 
where $R_{b}$ is the drop radius, $V$ is the impact velocity, and $\rho_{\ell}$ and $\mu_{g}$ are the density of the liquid and dynamic viscosity of the gas [4,14-19].

Interferometry can measure the air-layer profiles, but early studies were mostly limited to low impact velocities [20-22]. Total internal reflection (TIR) imaging can pin down the actual contacts [23], of particular importance in studying Leidenfrost impacts $[24,25]$. Here, the exact surface temperature [26] and the heat transfer between the drop and substrate and its cooling can be of primary importance [27].

The first time-resolved imaging of the air-disc dynamics [28] showed good agreement with the available theories, at atmospheric pressure [18], including the effects of compressibility of the gas at the higher impact velocities $V$, via the compressibility factor $\epsilon=P_{\mathrm{atm}} /\left(R V^{7} \rho_{\ell}^{4} / \mu_{g}\right)^{1 / 3}$ [4].

The experimental configuration is shown in Fig. 1 and is similar to that used in our previous work [12,28,29], though now the impacts are performed inside an acrylic vacuum chamber. The impact is viewed though the glass substrate, using long-distance microscopes with adjustable magnification and aperture, at pixel resolution down to $1.02 \mu \mathrm{m} / \mathrm{px}$. Figure 1(b) shows the transmission interferometric optical setup, which uses monochromatic red pulsed light of wavelength $\lambda_{\text {red }}=640 \mathrm{~nm}$. The rapid motions required observation with a high-speed video camera (Kirana-05M, Specialized Imaging, Tring UK) acquiring 180 frames at up to $5 \times 10^{6} \mathrm{fps}$ with a resolution of $924 \times 768$ px irrespective of the frame rate used. Video clips were acquired at several frame rates to capture the short- and long-term dynamics. The diode-laser pulse duration of 30-170 ns per frame minimized motion smearing. The precision of the interferometric thickness measurements is around $20 \mathrm{~nm}[12,30]$.

Drops of deionized water from a Milli-Q Academic were pinched off from a flat stainless steel nozzle $6 \mathrm{~mm}$ in diameter. The resulting drop was axisymmetric with an equivalent spherical diameter of $D \simeq 5.7 \mathrm{~mm}$. The release heights, measured from the nozzle to the solid surface, were varied from 7 to $136 \mathrm{~cm}$, giving impact velocities between 1.0 and $5.1 \mathrm{~m} / \mathrm{s}$. Using drops larger than the capillary length, the pinch-off produces significant drop oscillations [31]. The shape of the impacting drop was therefore monitored with a second high-speed video camera, viewing from the side, to provide the bottom radius of curvature at impact $R_{b}$ [28]. Gravity sets $V$, but is irrelevant to the rapid impact dynamics [32]. The effects of evaporation will, of course, depend on the volatility of the liquid. This issue was raised with regards to the study of Xu et al. [3] who used ethanol to form their drops [34,35]. Water, which is less volatile than ethanol, is used in our experiments; over the entire range of ambient pressures investigated, we estimate the effects of evaporation to be negligible [32].

Figure 2 shows a series of air discs for different ambient pressures $P$, while the impact velocity is kept constant.

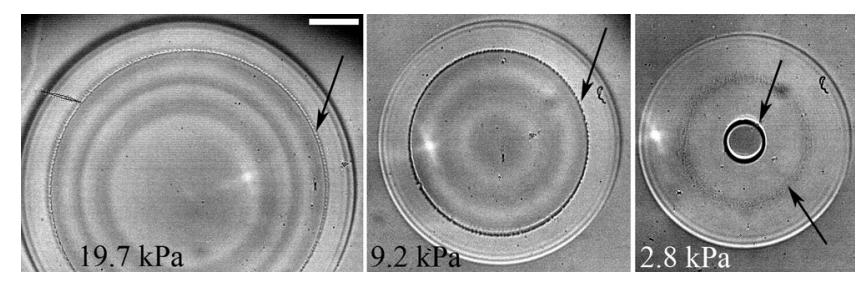

FIG. 2. Reduction in the size of the air disc as the ambient pressure is lowered, while keeping the water-drop impact velocity constant, $V=2.3 \mathrm{~m} / \mathrm{s}$. From left to right, $P=19.7,9.2$, and $2.8 \mathrm{kPa}, R_{b}=4.10,3.57$, and $3.80 \mathrm{~mm}, \mathrm{St}=1.99 \times 10^{-6}$, $2.28 \times 10^{-6}$, and $2.14 \times 10^{-6}$, and $\epsilon^{-1}=21,43$, and 144. The frames are shown, from the separate video clips, at $t=0.6,0.8$, and $1.4 \mu \mathrm{s}$ after first contact. The upper arrows mark the contact line. The lower arrow in the last panel points at a faint ring of microbubbles. These impacts are onto molecularly smooth freshly cleaved mica surfaces. The scale bar is $100 \mu \mathrm{m}$ long.

Both the thickness and width of the disc reduce rapidly with lowering $P$. In the images, the number of fringes within the disc shows that its central thickness $H^{*}$ reduces from $1.12 \mu \mathrm{m}\left(3.5 \times \lambda_{\text {red }} / 2\right)$ to $0.8 \mu \mathrm{m}$ and in the third panel to less than $160 \mathrm{~nm}$. The disc radius at first contact $L_{0}$ reduces here correspondingly from 288 to 191 and $51 \mu \mathrm{m}$. At the lowest $P$ there appears a faint ring of microbubbles (lower arrow) at a larger radius. At slightly higher $P=$ $4.3 \mathrm{kPa}$ a novel phenomenon appears, as shown in Fig. 3. It shows a time sequence of an impact with a double contact between the drop and substrate. It first entraps a central air disc followed by a second outer contact to trap a circular band of air.

The outer contact occurs $\simeq 800 \mathrm{~ns}$ after the first one. The central air disc contracts into a bubble in $18 \mu$ s. The toroidal film develops prominent disturbances on its inner edge with smaller disturbances on the outer edge. The band contracts and breaks up into numerous bubbles. The outer edge of the first contact is marked by a ring of microbubbles, while the outermost contact shows only a faint ring (arrow in last panel), as is expected for a smooth surface [29]. Based on the air volume in the resulting bubbles, we can estimate the initial uncompressed thickness of the central disc as $800 \mathrm{~nm}$ and the toroidal band as $600 \mathrm{~nm}$. Keep in mind that the lack of interference fringes within the disc $\left(H^{*}<160 \mathrm{~nm}\right)$ can be explained by large compression of the gas during the early stages of this impact, also suggesting a compression ratio of more than $4[22,28]$.

Figure 5 shows that the radial size of the air disc bifurcates at a value of $\epsilon^{-1} \simeq 25$. This bifurcation is indeed imprinted on the same impact, i.e., double contacts. The radius of the second contact, as well as the outer radius of the band of microbubbles, follows the same trend as the data prior to the bifurcation, see filled markers in Fig. 5. The outer edge of the first contact is marked by a layer of microbubbles. This band of microbubbles becomes much wider for the glass surfaces, compared to the molecularly smooth mica surface. Figure 4 shows two such realizations 


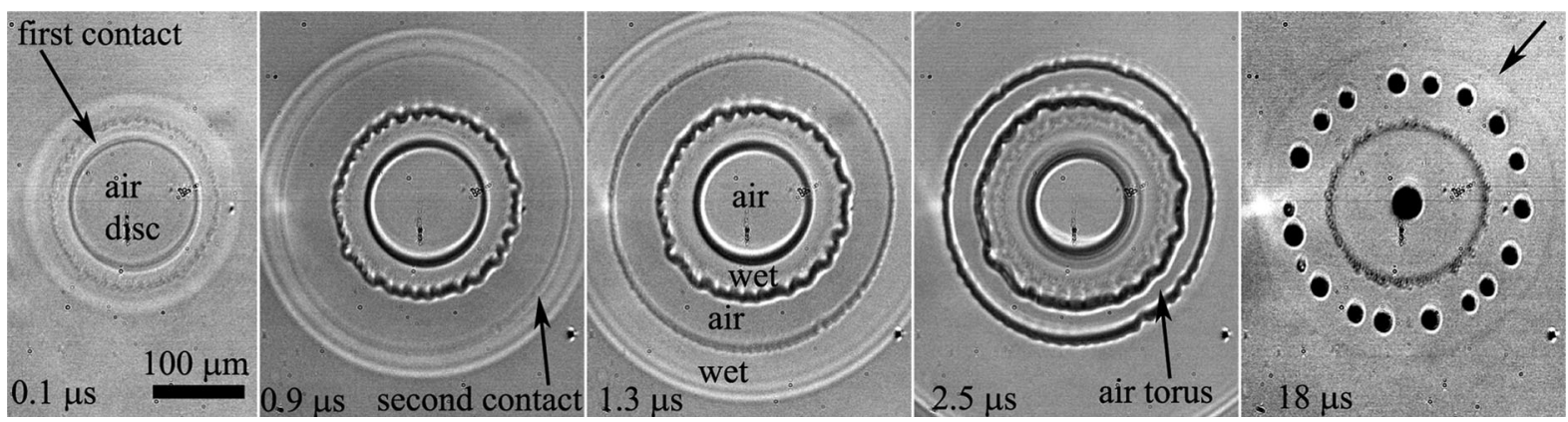

FIG. 3. Double contact, for a water drop impacting on a freshly cleaved mica sheet, at $P=4.3 \mathrm{kPa}, V=2.27 \mathrm{~m} / \mathrm{s}, R_{b}=3.66 \mathrm{~mm}$, $\epsilon^{-1}=93$, and $\mathrm{St}=2.22 \times 10^{-6}$. Frames are shown at times relative to the first contact, $t \simeq 0.1,0.9,1.3,2.5$, and $18 \mu \mathrm{s}$. The scale bar is $100 \mu \mathrm{m}$ long.

for impacts onto two brands of microscope slides. This may be related to local contacts, as observed in [29]. Keep in mind that these glass surfaces are exceedingly smooth, with

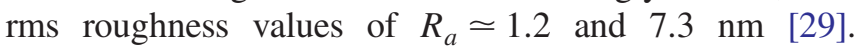
Figure 5 contains measurements for all three types of surfaces, glass with minute roughness as well as the mica molecularly smooth surface. The surface type modifies the structure of the bubbles and details of the contact [32], but does not alter its overall nature, suggesting the double contact has to do with the dynamics of the air layer before the molecular contact.

We note that this double-contact regime has not been predicted in any of the multitude of theoretical studies on the subject. Because of the fact that the outer contact size reduces continuously through the bifurcation, we conclude that new dynamics set in at this value of the compressibility factor, to promote the earlier inner contact. New effects which set in at very thin air layers are rarefied gas effects and possibly van der Waals forces. The Knudsen number $\mathrm{Kn}=\lambda_{0} / H^{*} \simeq \lambda_{0} /\left(R_{b} \mathrm{St}^{2 / 3}\right)$, characterizes the importance of rarefied-gas effects. Here the mean-free path in the gas at ambient conditions $\lambda_{0}$ is proportional to $T_{0} / P_{0}$ where $P_{0}$ and $T_{0}$ are the ambient gas pressure and temperature [36].
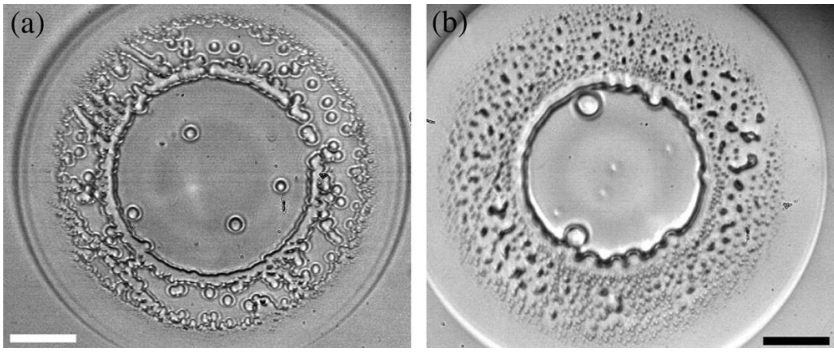

FIG. 4. (a) Extended band of bubbles outside the first contact, for impact on Fisher glass, rms surface roughness $R_{a}=1.2 \mathrm{~nm}$ [29]. Shown at $t=1.2 \mu \mathrm{s}, \quad V=2.3 \mathrm{~m} / \mathrm{s}, \quad P=10.2 \mathrm{kPa}$, $\mathrm{St}=2.02 \times 10^{-6}$, and $\epsilon^{-1}=40$. (b) Band of microbubbles for impact on Corning glass, with $R_{a}=7.3 \mathrm{~nm}$ [29], shown at $t=2.8 \mu \mathrm{s} . V=1.5 \mathrm{~m} / \mathrm{s}, P=3.5 \mathrm{kPa}, \mathrm{St}=2.74 \times 10^{-6}$, and $\epsilon^{-1}=43$. Scale bars are $100 \mu \mathrm{m}$ long.
At standard atmospheric conditions $\lambda_{0}=70 \mathrm{~nm}$. Assuming ideal gas behavior, the local mean-free path $\lambda=\lambda_{0} \rho_{0} / \rho_{g}$, for gas density $\rho_{g}$. Figure 6 shows where our impact conditions fall on a regime diagram, constructed for a typical droplet size in our experiment of $R_{b}=4 \mathrm{~mm}$. It shows that double contacts occur within the rarefied gas regime, where $\mathrm{Kn} \gtrsim 1$, indicating significant rarefied gas effects. Physically these effects include a pronounced Navier slip along the surfaces bounding the gas layer. With Navier slip, the axial gas mass flux is proportional to $h^{3}+6 \lambda h^{2}$, where $h$ is the local gas film height. Consequently, Navier slip enhances the rate at which gas can escape the oncoming droplet and reduces the volume trapped.

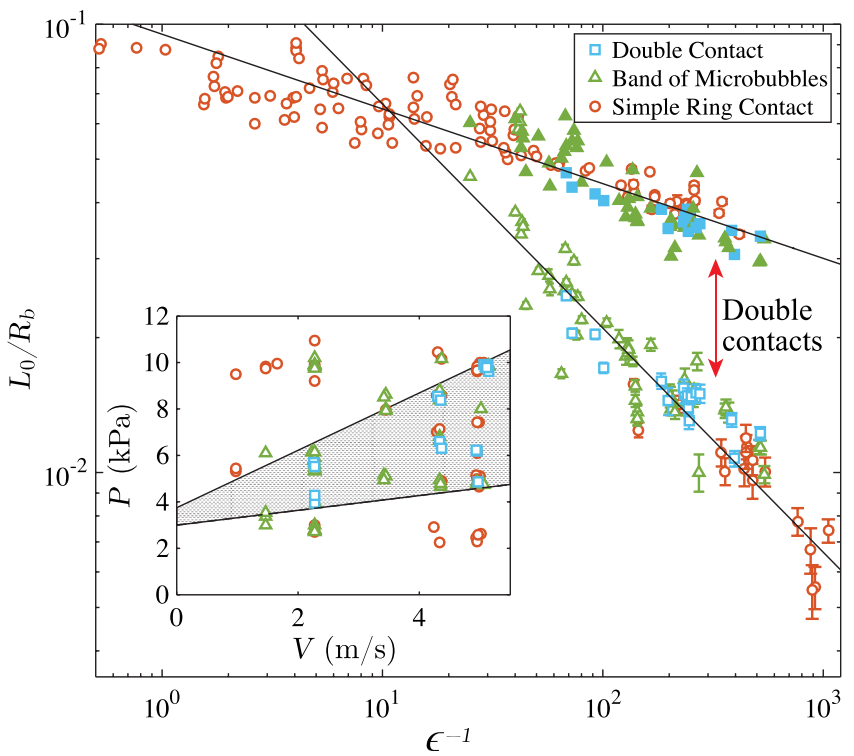

FIG. 5. The radial extent of the air disc vs the compressibility factor. The steeper curve has a slope of $-1 / 2$ and the shallower curve has a slope of $-1 / 6$. For the cases where double contact or bands of microbubbles were observed, the filled markers indicate the radial extent of the second contact or the outer edge of the band of microbubbles. The inset shows the pressure band (shaded area) where double contacts were observed, for water drops of $R_{b} \sim 3 \mathrm{~mm}$. 


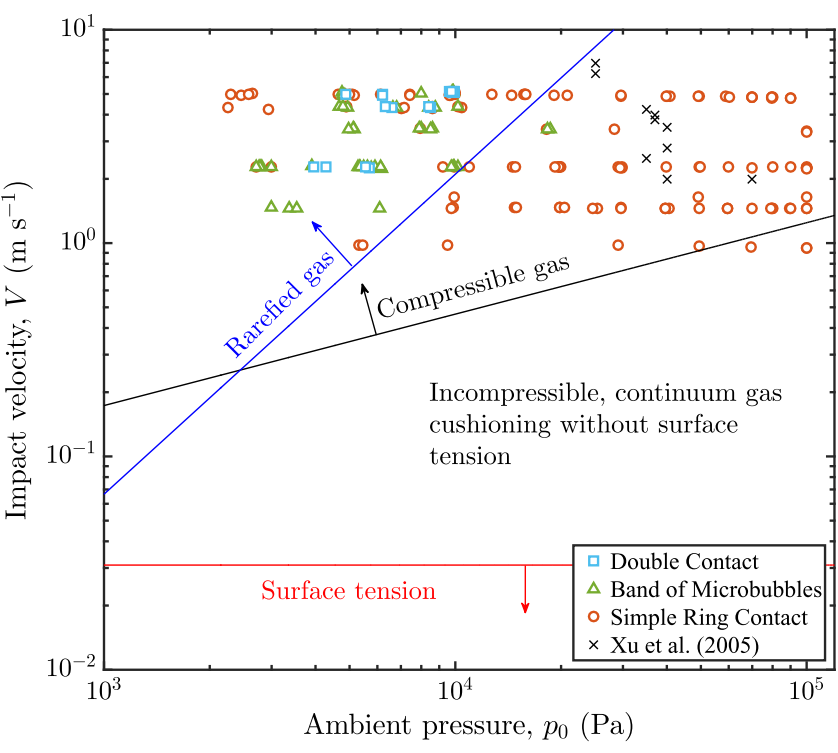

FIG. 6. Regime map showing the important dynamics for each region for a typical water drop radius $(4 \mathrm{~mm})$ used in our experiments. Experimental data has been overlaid with the markers indicating which type of contact was observed. Note that all of the observed double contacts and the majority of bands of microbubbles occur within the rarefied gas regime. The transition to the compressible regime occurs for $\epsilon^{-1} \gtrsim 1$ (black line), and the transition to the rarefied gas regime occurs for $\mathrm{Kn} \gtrsim 1$ (blue line). Surface tension is only significant for $V \lesssim\left(\mu_{g} \sigma^{3} / \rho_{l}^{4} R_{b}^{4}\right)^{1 / 7}$ (red line) [20]. Data from $\mathrm{Xu}$ et al. [3] are also included for comparison.

For a nonrarefied compressible gas, the scaling argument of Mandre et al. [4] predicts an initial disc radius $L_{0} / R_{b} \mathrm{St}^{1 / 3} \sim \epsilon^{(2-\gamma) /(4 \gamma-2)}$. Note that although this relation yields exponents of $1 / 6$ and $1 / 2$ for adiabatic and isothermal compression, respectively, that this does not correspond to the power laws shown in Fig. 5 as the figure is scaled differently. For an incompressible rarefied gas, Duchemin and Josserand [36] find $L_{0} / R_{b} \mathrm{St}^{1 / 3} \sim \mathrm{Kn}^{-1}$. In order to obtain a scaling for the initial disc radius for a droplet with an initially undisturbed free surface $h=r^{2} / 2 R_{b}-V t$, cushioned by a compressible, rarefied gas, we must consider a lubrication equation of the form

$$
12 \mu_{g}\left(\rho_{g} h\right)_{t}=\nabla_{r} \cdot\left[\rho_{g}\left(h^{3}+6 \lambda h^{2}\right) \nabla_{r} P_{g}\right]
$$

Here $\nabla_{r}$ is the axial gradient operator in the plane of the gas film. The equation of state for the gas is $P_{g}=P_{0}\left(\rho_{g} / \rho_{0}\right)^{\gamma}$, with $\gamma=1$ for isothermal and $\gamma=(7 / 5)$ for adiabatic compression. The normal stress balance gives a pressure at the droplet interface $P_{l}=P_{g}+\sigma \nabla_{r}^{2} h$, where $\sigma$ is the surface tension. Our experimental data lie outside the region where surface tension is important (Fig. 6), and, consequently, the free-surface acceleration can be related to the gas pressure through $\rho_{\ell} h_{t t}=\mathcal{H}\left[\nabla_{r} P_{g}\right]$. In two dimensions,
$\mathcal{H}$ is a Hilbert transform [14] and is given by Hicks et al. [17] for axisymmetry.

To deform the droplet, its free surface must be brought to rest over a time scale $\tau \sim H / V$, while the axial length scale $L=\sqrt{R_{b} H}$, where $H$ is the gas film height. In the rarefied gas regime $H \ll \lambda$, and so Eq. (1) implies $P_{g} \sim \mu_{g} V R_{b} / \lambda H$, where $\lambda$ retains a dependence on $P_{g}$. The droplet deceleration also gives a pressure scale $P_{g} \sim \rho_{\ell} V^{2} R_{b}^{1 / 2} / H^{1 / 2}$. These two pressure scales are equal (giving rise to coupled interactions between droplet and gas pressure film), when $H=H^{*}=R_{b} \mathrm{St}^{2 / 3} \epsilon^{-2 /(\gamma-1)} \mathrm{Kn}^{-2 \gamma /(\gamma-1)}$. Gas compressibility sets in when $P_{g} \sim P_{0}$, and for a rarefied gas this occurs at height $H=H^{*}=R_{b} \mathrm{St}^{2 / 3} \epsilon^{-2(\gamma+1) /(2 \gamma+1)} \mathrm{Kn}^{-2 \gamma /(2 \gamma+1)}$. Below this height $\left(\rho_{g} h\right)_{t} \approx 0$, i.e., $H=\rho_{0} H^{*} / \rho_{g}$. The equation of state and the gas pressure scale based on the free-surface deceleration then imply the initial disc radius should scale as

$$
L_{0} / R_{b} \mathrm{St}^{1 / 3} \sim \epsilon^{1 /(2 \gamma+1)(2 \gamma-1)} \mathrm{Kn}^{-2 \gamma^{2} /(2 \gamma+1)(2 \gamma-1)} .
$$

For adiabatic conditions $(\gamma=1.4)$ this scaling with $\sim \epsilon^{0.15} \mathrm{Kn}^{-0.57}$ gives reasonable fit to the data, as shown in the Supplemental Material [32]. Agreement with the adiabatic condition is better than with the isothermal condition, indicating that the heat generated compressing the gas cannot be transferred to the surroundings on the short time scale of cushioning.

For rarefied gas impacts, both the scaling law for the incompressible gas limit [36] and that developed herein for compressible gas predict reductions in $L_{0}$ for increasing $\mathrm{Kn}$. For the lowest pressure ratio, these scaling laws suggest 30-fold (incompressible), 30-fold (isothermal), and tenfold (adiabatic) reductions in $L_{o}$, in reasonable accordance with Fig. 5.

How thin does the air disc become? For our largest $V=5.1 \mathrm{~m} / \mathrm{s}$, the fringes disappear at $P \simeq 50 \mathrm{kPa}$, indicating $H \simeq 160 \mathrm{~nm}$. For a further reduction in $P$ to $3 \mathrm{kPa}$, the incompressible rarefied-gas scaling law [36] predicts subnanometer-thickness gas layers, well into the van der Waals-dominated regime, supporting our proposal that slip from rarefied-gas effects can promote localized contacts.

Herein, we have investigated the effects of reduced pressure on the air entrapment under an impacting drop. Double contacts are a robust phenomenon, showing up over a range of $V$ (Fig. 5, inset) and even for impacts on a thin film of liquid [32]. It is difficult to disentangle the rarefaction and compression effects in our data, as they are both primarily adjusted by lowering $P$. A full theory is therefore needed, along with high-speed TIR imaging to resolve the thinnest air layers.

This study was supported by King Abdullah University of Science and Technology (KAUST) under Grant No. URF/1/ 2621-01-01. E. Q. L. acknowledges the Thousand Young 
Talents Program of China, the National Natural Science Foundation of China (Grants No. 11642019, No. 11772327, and No. 11621202) and Fundamental Research Funds for the Central Universities (Grant No. WK2090050041).

*sigurdur.thoroddsen@kaust.edu.sa

[1] A. L. Yarin, Annu. Rev. Fluid Mech. 38, 159 (2006).

[2] C. Josserand and S. T. Thoroddsen, Annu. Rev. Fluid Mech. 48, 365 (2016).

[3] L. Xu, W. W. Zhang, and S. R. Nagel, Phys. Rev. Lett. 94, 184505 (2005).

[4] S. Mandre, M. Mani, and M. P. Brenner, Phys. Rev. Lett. 102, 134502 (2009).

[5] S. T. Thoroddsen, T. G. Etoh, K. Takehara, N. Ootsuka, and Y. Hatsuki, J. Fluid Mech. 545, 203 (2005).

[6] S. T. Thoroddsen, K. Takehara, and T. G. Etoh, J. Fluid Mech. 706, 560 (2012).

[7] M. M. Driscoll and S. R. Nagel, Phys. Rev. Lett. 107, 154502 (2011).

[8] C. S. Stevens, A. Latka, and S. R. Nagel, Phys. Rev. E 89, 063006 (2014).

[9] G. Riboux and J. M. Gordillo, Phys. Rev. Lett. 113, 024507 (2014).

[10] R. C. A. van der Veen, T. Tran, D. Lohse, and C. Sun, Phys. Rev. E 85, 026315 (2012).

[11] J. de Ruiter, R. Lagraauw, D. van den Ende, and F. Mugele, Nat. Phys. 11, 48 (2015).

[12] K. Langley, E. Q. Li, and S. T. Thoroddsen, J. Fluid Mech. 813, 647 (2017).

[13] C. J. Howland, A. Antkowiak, J. R. Castrejón-Pita, S. D. Howison, J. M. Oliver, R. W. Style, and A. A. CastrejónPita, Phys. Rev. Lett. 117, 184502 (2016).

[14] F. T. Smith, L. Li, and G. X. Wu, J. Fluid Mech. 482, 291 (2003).

[15] A. A. Korobkin, A. S. Ellis, and F. T. Smith, J. Fluid Mech. 611, 365 (2008).

[16] P. D. Hicks and R. Purvis, J. Fluid Mech. 649, 135 (2010).
[17] P. D. Hicks, E. V. Ermanyuk, N. V. Gavrilov, and R. Purvis, J. Fluid Mech. 695, 310 (2012).

[18] P. D. Hicks and R. Purvis, J. Fluid Mech. 735, 120 (2013).

[19] L. Duchemin and C. Josserand, Phys. Fluids 23, 091701 (2011).

[20] W. Bouwhuis, R. C. A. van der Veen, T. Tran, D. L. Keij, K. G. Winkels, I. R. Peters, D. van der Meer, C. Sun, J. H. Snoeijer, and D. Lohse, Phys. Rev. Lett. 109, 264501 (2012).

[21] J. S. Lee, B. M. Weon, J. H. Je, and K. Fezzaa, Phys. Rev. Lett. 109, 204501 (2012).

[22] Y. Liu, P. Tan, and L. Xu, J. Fluid Mech. 716, R9 (2013).

[23] J. M. Kolinski, S. M. Rubinstein, S. Mandre, M. P. Brenner, D. A. Weitz, and L. Mahadevan, Phys. Rev. Lett. 108, 074503 (2012).

[24] T. Tran, H. J. J. Staat, A. Prosperetti, C. Sun, and D. Lohse, Phys. Rev. Lett. 108, 036101 (2012).

[25] M. Shirota, M. A. J. van Limbeek, C. Sun, A. Prosperetti, and D. Lohse, Phys. Rev. Lett. 116, 064501 (2016).

[26] V. Bertola, Int. J. Heat Mass Transfer 85, 430 (2015).

[27] M. A. J. van Limbeek, M. H. Klein Schaarsberg, B. Sobac, A. Rednikov, C. Sun, P. Colinet, and D. Lohse, J. Fluid Mech. 827, 614 (2017).

[28] E. Q. Li and S. T. Thoroddsen, J. Fluid Mech. 780, 636 (2015).

[29] E. Q. Li, I. U. Vakarelski, and S. T. Thoroddsen, J. Fluid Mech. 785, R2 (2015).

[30] J. de Ruiter, F. Mugele, and D. Van der Ende, Phys. Fluids 27, 012104 (2015).

[31] M.-J. Thoraval, K. Takehara, T. G. Etoh, and S. T. Thoroddsen, J. Fluid Mech. 724, 234 (2013).

[32] See Supplemental Material at http://link.aps.org/ supplemental/10.1103/PhysRevLett.119.214502 for further details on the effects of gravity and evaporation as well as supplemental figures, which includes Ref. [33].

[33] A. H. Persad and C. A. Ward, Chem. Rev. 116, 7727 (2016).

[34] K. Sefiane, Phys. Rev. Lett. 96, 179401 (2006).

[35] L. Xu, W. W. Zhang, and S. R. Nagel, Phys. Rev. Lett. 96, 179402 (2006).

[36] L. Duchemin and C. Josserand, C. R. Mech. 340, 797 (2012). 